\title{
Building Response due to Ground Vibration Simple Prediction Model Based on Experience with Detailed Models and Measurements
}

\author{
Lutz Auersch \\ Bundesanstalt für Materialforschung und - prufüng (BAM), D 12200 Berlin, Germany
}

(Received 21 September 2009; revised 12 April 2010; accepted 12 May 2010)

Construction work, such as pile driving and soil compaction, or road and railway traffic excite nearby buildings, and the perceptible or audible vibration can be a nuisance for nearby inhabitants. A simplified building model has been created for these situations, which includes the effects of soil-structure interaction, the low-frequency amplification along the height of the building as well as the high-frequency reduction and the floor resonances. The model consists of one wall for all supporting structures (walls and columns) and one floor for each storey. The effect of different floor resonance frequencies is included in a stochastic procedure. The soil is modelled by a spring and a viscous damper, and the free-field amplitudes of the soil are applied under this soil element.

The model can be calculated by transfer matrices or in a continuous wave-type version where an analytical solution can be evaluated numerically. The building response in the high-frequency (acoustic) region is calculated as mean values over wider frequency bands. The approach to an infinite building model can be found for these high frequencies and the corresponding soil-structure transfer can be described by the ratio of impedances at foundation level.

The rules for choosing the parameters to obtain realistic results are derived from complex calculations - for example, for the stiffness and damping of building foundations - and many measurements as for the damping of floor resonances. The influences on the floor resonance from the soil (damping) and the supporting structure (detuning) are important. Some more effects will be discussed by the simplified and detailed models and by measurements to establish a good understanding of ground-induced building vibrations.

\section{INTRODUCTION}

The problem of ground-borne building vibration is shown in Fig. 1a. Train-induced vibrations propagate through the soil and excite neighbouring buildings. The problem is divided into emission (excitation by railway traffic), transmission (wave propagation through the soil), and immission (transfer into a building) of vibration. The link between the modules is made by the excitation force between emission to transmission and by the free-field vibration between transmission and immission. The free-field vibration is the vibration at the place of the building in the case that the building is not there. Note that the problem and not the physical system is cut into three parts. Emission and immission is well analysed, including the track-soil and building-soil interaction.

Prediction software ${ }^{1}$ that includes the three modules for emission, ${ }^{2}$ transmission, ${ }^{3}$ and immission ${ }^{4}$ has been prepared. The user-friendly software combines simple models that are derived from the results of complex models and from experimental experience. Simple models are necessary for a userfriendly prediction software so that it only takes a short time on the computer and, most important, so that there is a simple input procedure with only a few relevant parameters.

This article is concerned with immission, the third part of the prediction scheme. Immission is the vibration transfer from the free-field of the soil to the different building elements. The model presented here is part of the prediction software. The basic concept of the prediction of ground-borne building vibration, the simple wall model, was already developed and analysed by the 1980s. ${ }^{5}$ Experimental and numerical experience with floor resonances ${ }^{6}$ was used to build a practicable floorwall-soil model. ${ }^{4}$ In the last decade, about 10 specific building projects have been treated by detailed laborious finite-element models. The complex building models, as shown in Fig. 2 are also used for parametric studies and an improved understanding of building vibrations.

The basic concepts of building vibration are mainly developed for earthquake problems ${ }^{7}$, as well as the concepts for the dynamic soil-structure interaction. ${ }^{8}$ Technically induced building vibrations are treated in Bachmann\&Ammann ${ }^{9}$, whereas the wave propagation through building structures and the more statistical concepts for higher frequencies can be found in Cremer, et al. ${ }^{10}$ Articles about ground-borne building vibration are mostly about singular projects. Recently a 3-storey office building ${ }^{11}$ and a 2 -storey residential building ${ }^{12}$ have been calculated by a complete detailed model. More general rules have been searched and found for elementary building structures. ${ }^{5,13-17}$ There are two more $\mathrm{PhD}$ theses dealing with ground-borne building vibration where Breitsamter developed a number of simplified models for different building types and structural elements ${ }^{18}$, and some more calculated projects and measurements of the Federal Institute for Materials Research and Testing (BAM) are given in Meinhardt. ${ }^{19}$ Experimental experience of this subject (measurement results of several buildings) is presented in Nelson and Saurenman, Jacobson, and especially by Watts, who presented a number of research reports about road traffic induced building vibration. ${ }^{20-22}$

In this contribution, all aspects of ground-borne building vibration are discussed by a simple but complete building model 